\title{
Neutral delay differential equations: An improved approach and its applications in the oscillation theory
}

\author{
Osama Moaaz ${ }^{1}$, George Chatzarakis ${ }^{2}$, and Ali Muhib ${ }^{3}$ \\ ${ }^{1}$ Mansoura University Faculty of Science \\ ${ }^{2}$ School of Pedagogical and Technological Education \\ ${ }^{3} \mathrm{Ibb}$ University
}

August 25, 2020

\begin{abstract}
The objective of this study is to establish new sufficient criteria for the oscillation of the 2nd-order neutral equation $\$ \backslash$ left( $\mathrm{r} \backslash \operatorname{left}\left(\mathrm{z}^{\wedge}\{\backslash\right.$ prime $\} \backslash$ right $) \wedge\{\backslash$ alpha $\} \backslash$ right $)^{\wedge}\{\backslash$ prime $\} \backslash \operatorname{left}(\mathrm{t} \backslash$ right $)+\mathrm{q} \backslash$ left $(\mathrm{t} \backslash$ right $) \mathrm{x}^{\wedge}\{\backslash$ beta $\} \backslash \operatorname{left}(\backslash$ sigma $\backslash$ left $(\mathrm{t} \backslash$ right $)$ $\backslash$ right $)=0, \$$ where $\$ \mathrm{t} \backslash$ geq $\mathrm{t}_{-}\{0\} \$$ and $\$ \mathrm{z} \backslash \operatorname{left}(\mathrm{t} \backslash$ right $)=\mathrm{x} \backslash \operatorname{left}(\mathrm{t} \backslash$ right $)+\mathrm{px} \backslash \operatorname{left}(\backslash$ tau $\backslash \operatorname{left}(\mathrm{t} \backslash$ right $) \backslash$ right $) \$ \%$. We improve the known criteria by establishing a new relationship between the solution $\$ \mathrm{x} \$$ and the corresponding function $\$ \mathrm{z} \$$. To show the importance of our results, we provide two examples.
\end{abstract}

\section{Hosted file}

Moaaz_7.pdf available at https://authorea.com/users/353711/articles/477487-neutral-delaydifferential-equations-an-improved-approach-and-its-applications-in-the-oscillationtheory 